УДК 004.932

Дата подачи статьи: 24.11.17

DOI: $10.15827 / 0236-235 X .122 .362-367$

2018. Т. 31. № 2. С. 362-367

\title{
СОЗДАНИЕ ПАНОРАМНЫХ АЭРОФОТОСНИМКОВ С ИСПОАЬЗОВАНИЕМ КВАДРОКОПТЕРА
}

\author{
А.И. Пахирка ${ }^{1}$, к.т.н., доиент, pahirka@sibsau.ru \\ A.Г. Зотин 1, к.т.н., доиент, zotinkrs@gmail.com \\ В.В. Буряченко 1, к.т.н., доиент, buryachenko@sibsau.ru
}

1 Сибирский государственный университет науки и технологий им. академика М.Ф. Решетнева, просп. им. газеты "Красноярский рабочий", 31, г. Красноярск, 660037, Россия

В статье рассматривается подход к формированию панорамных снимков, представляющих высокодетализированные изображения местности. Для получения панорамных изображений высокого качества требуются специализированное оборудование и по возможности максимальная высота съемки. Эти факторы можно исключить, если использовать сшивку изображений, получаемых с камер, расположенных на недорогих беспилотных летательных аппаратах - квадрокоптерах. Одним из подходов к формированию панорамных снимков является использование серии изображений или видеопоследовательности, полученных при съемке с квадрокоптера или иного беспилотного летательного аппарата.

Для сшивки изображений применяются методы сопоставления точечных особенностей, при этом алгоритмы обнаружения особых точек должны гарантировать инвариантность относительно любых преобразований изображения для того, чтобы можно было выполнить анализ данных и формирование панорамных снимков. Для исследования были выбраны такие алгоритмы, как FAST, FAST-ER и SURF, поскольку на текущий момент они являются наиболее распространенными для решения подобного рода задач. В качестве алгоритмов для определения соответствий найденных точечных особенностей были выбраны алгоритмы RANSAC и MLESAC.

В работе предложен алгоритм создания панорамного аэрофотоснимка из набора последовательных изображений местности, полученных с беспилотного летательного аппарата, на основе сопоставления ключевых особенностей. Используется глобальное выравнивание панорамного снимка с применением аффинных преобразований. Особое внимание уделяется сшивке изображений с применением технологии многополосного смешивания, что обеспечивает качественную визуализацию в местах сшивки панорамного снимка.

Ключевые слова: детекторы особых точек, FAST, SURF, панорамный аэрофотоснимок.

В последние годы широко применяются такие устройства, как квадрокоптеры и беспилотные летательные аппарать (БПЛА) - дроны, активно используемые для съемки на малых высотах. Они находят применение в сфере удаленного мониторинга объектов и в обследовании сооружений, а также при оценке состояния земель. Одной из их задач является формирование панорамных снимков - высокодетализированных изображений местности, которые зачастую невозможно получить в виде цельной фотографии [1].

Создание композитного снимка из последовательных изображений, полученных с БПЛА, является частным случаем построения панорамного снимка из набора произвольных изображений заданной местности. Условия задачи таковы, что необходимо построить последовательность планарных изображений в соответствии с траекторией полета БПЛА. Линейка БПЛА представлена широким классом устройств с различными летными и нагрузочными характеристиками, причем, чем ниже такие характеристики, тем БПЛА более подвержен влиянию воздушных потоков, что приводит к сложным условиям съемки, а в результате к получению размытых кадров. Напротив, бюджетная легковесная съемочная аппаратура означает получение изображений низкого разрешения. Разрабатываемый алгоритм включает отбор изображений, их локальное совмещение, глобальное выравнива- ние сцены и устранение визуальных артефактов в местах сшивки, что позволяет устранить указанные недостатки.

\section{Метод создания панорамного аэрофотоснимка}

Сведения о траектории полета БПЛА позволяют определить, какие изображения должны быть задействованы на текущем этапе создания панорамного снимка (рис. 1). Примем, что моменты начала и окончания съемки с целью получения панорамного снимка указываются пользователем. Строится граф пронумерованных изображений, и на текущем этапе создания панорамного снимка используются все имеющиеся соседние изображения. Такая процедура позволяет избавиться от дорогостоящего сопоставления случайных пар неотсортированных изображений [2].

Также следует проводить обязательную проверку на размытость изображений для ее устранения или удаления размытого изображения из набора сшиваемых изображений (при сильной степени размытости). В работе [3] предложен усовершенствованный метод устранения размытия, позволяющий оценивать четкость изображения на основе градиентной информации по всему текущему изображению относительно имеющихся изображений и заданному порогу размытия. Нахождение 


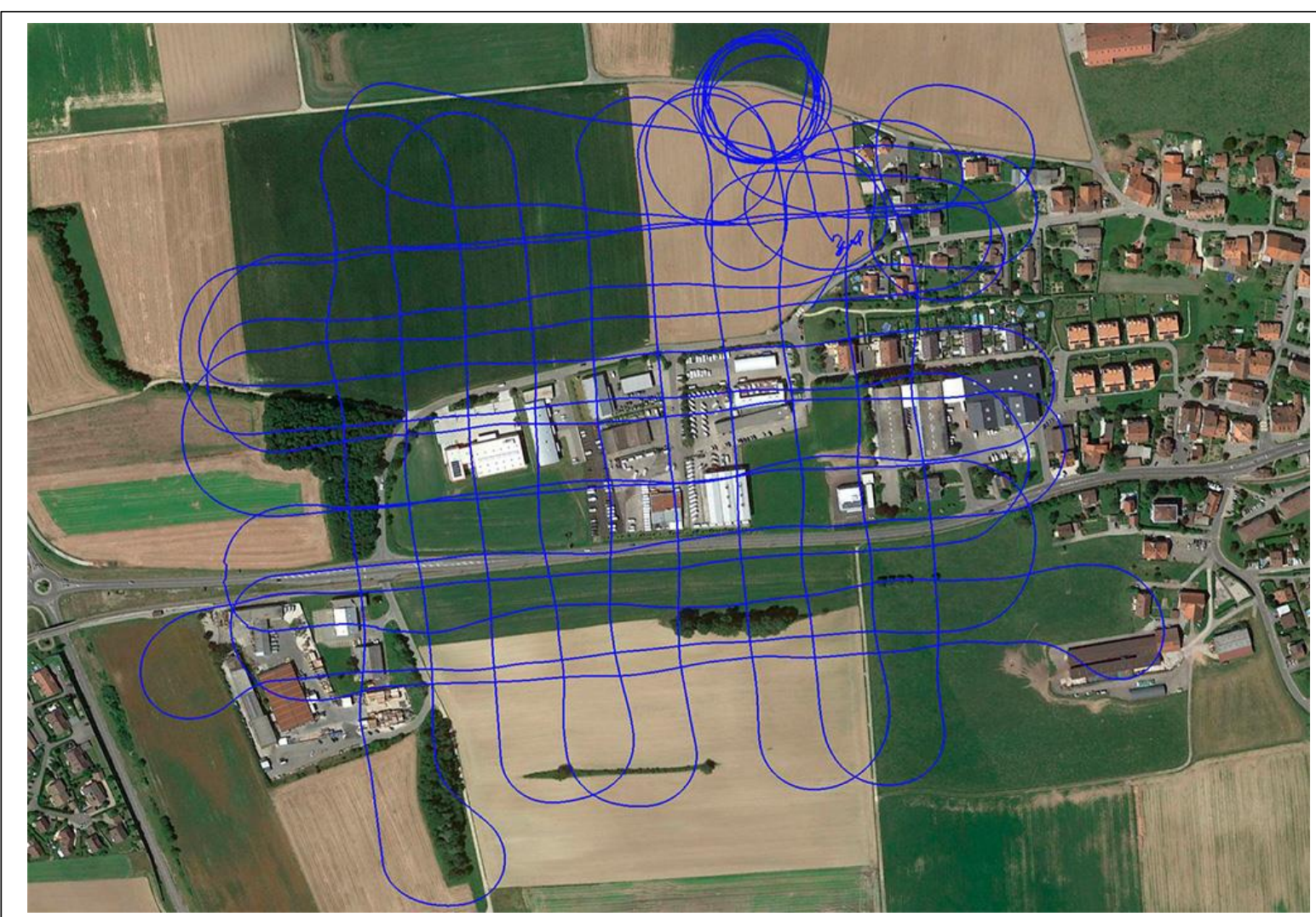

Pис. 1. Траектория движения БПЛА на Google Maps

Fig. 1. The trajectory of UAV movement on Google Maps

границ объектов на изображении выполняется оператором Собела. Для детализированных регионов следует применять анизотропный фильтр Гаусса для сглаживания негативных эффектов размытия, при этом сохраняя информацию о границах в неизменном виде. Используется анизотропный фильтр Гаусса с переменным ядром. Для гладких регионов применяется алгоритм нерезкого маскирования, основанный на вычитании размытого изображения из четкого изображения. В результате формируется изображение с частично или полностью устраненными эффектами размытия.

Совмещение изображений, которые в общем случае получены при различных углах наклона камеры, является одной из основных процедур создания панорамного снимка. Известны два основных подхода: непосредственное совмещение изображений и совмещение с использованием точечных дескрипторов [4]. Считается, что первый подход обеспечивает большую точность совмещения, однако имеет ограничения по параметрам съемки (они должны быть практически такими же, как параметры съемки исходного снимка). Более общим является подход на основе точечных дескрипторов (Speeded-Up Robust Feature (SURF) [5], Features from Accelerated Segment Test (FAST) [6], FAST Enhanced Repeatability (FAST-ER)) или стабильных областей (Affine Invariant Intensity Extrema, Maximally Stable Extremal Region (MSER)) [7]. В таком случае формирование панорамных изображений будет включать следующие основные этапы: обнаружение особых точек кадров видеопоследовательности и формирование наборов точечных особенностей, сопоставление этих наборов, построение проективного преобразования для выравнивания изображений и их переноса в общую плоскость, непосредственная сшивка выровненных изображений.

Помимо классических подходов, в последние годы развиваются быстрые методы построения панорамных снимков, например, основанные на расширенном фильтре Калмана и методе одновременной локализации и сопоставления (Simultaneous Localization and Mapping (SLAM)) [8].

В данной работе параллельно рассчитываются быстрые дескрипторы FAST и FAST-ER [9], предназначенные для обнаружения углов, и дескриптор SURF, учитывающий фактор масштабирования. Данные алгоритмы могут применяться для решения проблемы вычислительной сложности слияния изображений. В дескрипторе FAST проверяется, является ли пиксел $p$ углом, путем сравнения значения его яркости со значениями яркостей 16 пикселов $\left\{x_{1}, x_{2}, \ldots, x_{16}\right\}$ с радиусом 3 вокруг проверяемого пиксела $p$ (рис. 2).

Для ускорения работы алгоритма вначале рассматриваются пикселы с номерами 1, 5, 9 и 13. Если, по крайней мере, три из четырех пикселов 


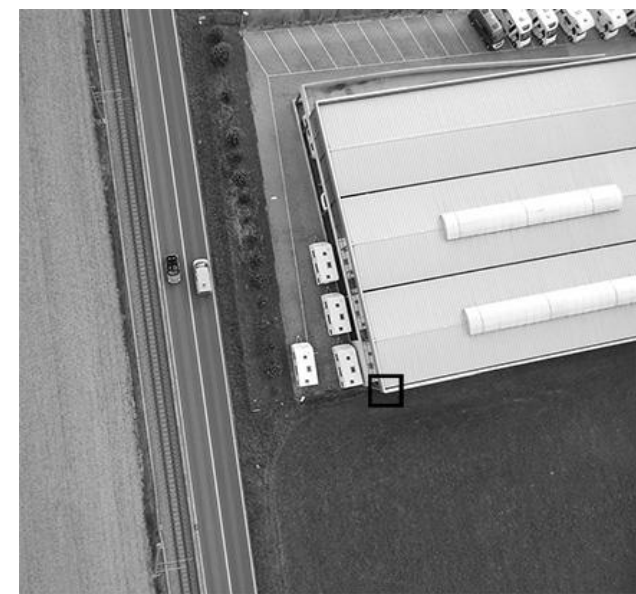

a)

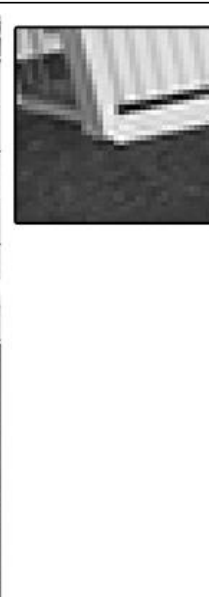

б)

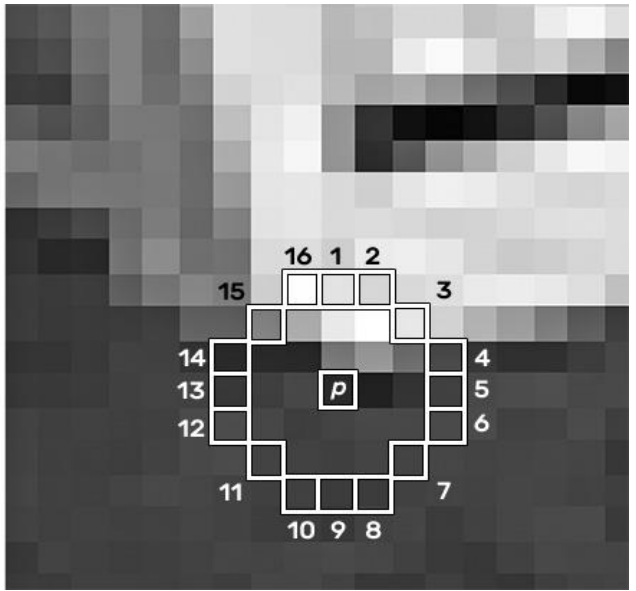

в)

Рис. 2. Нахождение углов: а) исходное изображение, б) увеличенный фрагмент, в) детектор FAST

Fig. 2. Corner detection: a) original image, б) enlarged fragment, в) FAST detector

удовлетворяют пороговому критерию, пиксел $p$ считается углом. Если хотя бы три из четырех значений находятся ниже порогового значения $I_{p}+T_{F A S T}$, пиксел $p$ не является точкой интереса и отбрасывается. В противном случае проверяются все 16 значений окружающих пикселов в виде вектора $S_{p \rightarrow x}$, принимающего соответственно темное, подобное и светлое значения в соответствии со следующим выражением:

$$
S_{p \rightarrow x}=\left\{\begin{array}{l}
d, I_{p \rightarrow x} \leq I_{p}-T_{F A S T}, \\
s, I_{p}-T_{F A S T}<I_{p \rightarrow x}<I_{p}+T_{F A S T}, \\
b, I_{p}+T_{F A S T} \leq I_{p \rightarrow x},
\end{array}\right.
$$

где $I_{x}$ - яркость пиксела на окружности вокруг проверяемого пиксела; $I_{p}$ - яркость проверяемого пиксела; $T_{F A S T}$ - пороговое значение для дескриптора FAST, например, $20 \%$ значения яркости.

Далее строятся подмножества $P_{d}, P_{s}, P_{b}$ и определяется переменная $K_{p}$, которая в соответствии с классификатором дерева решений [10] определяет, является ли проверяемый пиксел углом или нет.
Для снижения влияния шумов на результат работы алгоритма перед его применением рекомендуется произвести размытие изображения фильтром Гаусcа.

Для идентификации особой точки в дескрипторе SURF строится массив из 64 (в расширенной версии 128) чисел. При этом размер окна для поиска дескриптора равен $20 s$, где $s$ - масштаб, в котором была найдена особая точка. Дескриптор содержит описание градиентов для 16 квадрантов вокруг особой точки. Далее квадрат разбивается на 16 более мелких квадрантов. В каждом квадранте берется регулярная сетка $5 \times 5$, и для каждой точки сетки ищется градиент с помощью фильтра Хаара. Размер фильтра Хаара берется равным $2 s$ и для первой октавы составляет $4 \times 4$. Следует отметить, что при расчете фильтра Хаара изображение не поворачивается и фильтр рассчитается в обычных координатах изображения. Затем полученные координаты градиента $(d X, d Y)$ поворачиваются на угол, соответствующий ориентации квадрата. На рисунке 3 представлен пример обнаружения особых

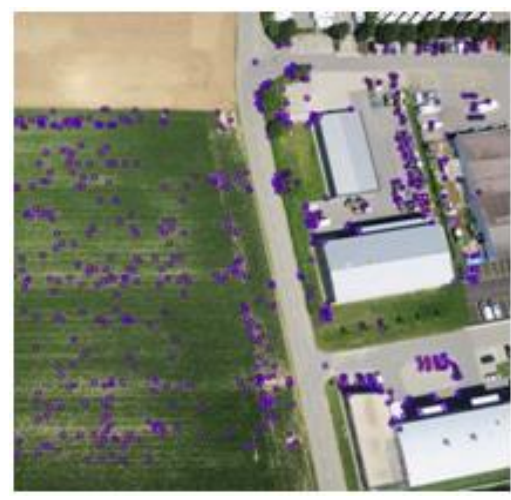

a)

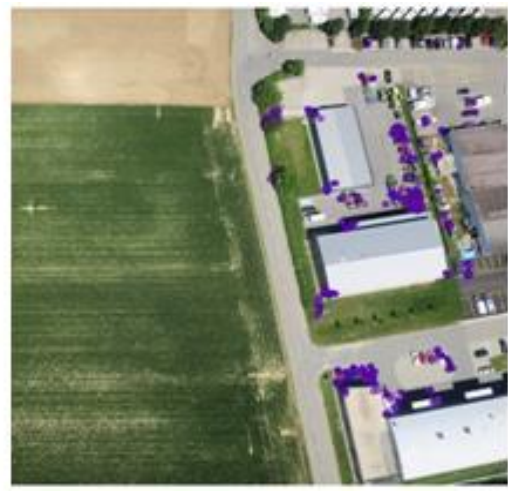

б)

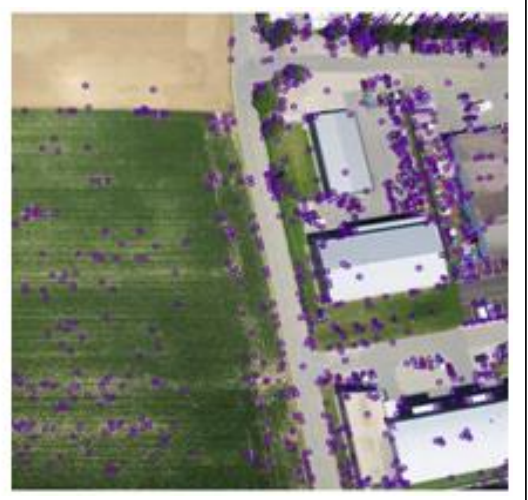

в)

Puc. 3. Нахождение особых точек: а) алгоритм FAST, б) алгоритм FAST-ER, в) алгоритм SURF

Fig. 3. Feature points detection: a) FAST algorithm, б) FAST-ER algorithm, в) SURF algorithm 
точек на изображении с применением алгоритмов FAST, FAST-ER и SURF [11].

Также для описания точки используется знак следа матрицы Гессе. Для светлых точек на темном фоне след отрицательный, а для темных точек на светлом фоне след положительный. Таким образом, SURF различает светлые и темные пятна.

Одним из самых распространенных способов оценки параметров модели является алгоритм RANSAC (RANdom SAmple Consensus) [12]. Концепция данного алгоритма основана на разделении всех исходных данных на выбросы (outliers) и попадания (inliers). Выбросами являются ложные точки, шумы, случайные включения в исходные данные, то есть такие точки, на основании которых нельзя построить требуемую модель. Попадания это точки, удовлетворяющие модели. Данный алгоритм выполняется итеративно. У алгоритма RANSAC имеется ряд недостатков, которые иногда не позволяют получить требуемый результат за приемлемое время, так как отсутствует верхняя граница времени, необходимого для вычисления параметров модели. В настоящее время разработаны, реализованы и протестированы модификации данного алгоритма. Одной из них является алгоритм MLESAC [13]. Данный алгоритм вычисляет вероятность гипотезы, представляя распространение ошибки как смешивание моделей. Для одновременного вычисления параметров модели и доли выбросов применяется схема максимизации ожидания. Для сшивки изображений требуется подобрать четыре наилучших сопоставления точечных особенностей, координаты которых используются для вычисления коэффициентов матрицы гомографии:

$$
\left.\left\lfloor\begin{array}{l}
x_{j} \\
y_{j} \\
1
\end{array}\right\rfloor=\left\lfloor\begin{array}{lll}
h_{1} & h_{2} & h_{3} \\
h_{4} & h_{5} & h_{6} \\
h_{7} & h_{8} & h_{9}
\end{array}\right\rfloor \mid \begin{array}{l}
x_{i} \\
y_{i} \\
1
\end{array}\right\rfloor,
$$

где $h_{1}, \ldots, h_{9}$ - коэффициенты матрицы; $\left(x_{i}, y_{i}\right)$ и $\left(x_{j}, y_{j}\right)$ - координаты соответствующих точек.

При проективном преобразовании, $h_{9}=1$, выражение (2) принимает вид

$$
x_{j}=\frac{h_{1} x_{i}+h_{2} y_{i}+h_{3}}{h_{7} x_{i}+h_{8} y_{i}+1}, \quad y_{j}=\frac{h_{4} x_{i}+h_{5} y_{i}+h_{6}}{h_{7} x_{i}+h_{8} y_{i}+1} .
$$

Тогда пара соответствующих точек связана следующими соотношениями:

$$
\begin{aligned}
& h_{1} x_{i}+h_{2} y_{i}+h_{3}-h_{7} x_{i} x_{j}-h_{8} x_{j} y_{i}=x_{j}, \\
& h_{4} x_{i}+h_{5} y_{i}+h_{6}-h_{7} x_{i} y_{j}-h_{8} y_{i} y_{j}=y_{j} .
\end{aligned}
$$

Сшивка изображений на основе матриц гомографии позволяет осуществлять локальное выравнивание панорамного снимка. Для глобального выравнивания должны одновременно использоваться все матрицы гомографии, что невозможно выполнить в реальном времени. В этом случае можно воспользоваться частичным выравниванием сшитых изображений на основе аффинных преобразо- ваний с использованием метода наименьших квадратов или применить эвристические алгоритмы поиска геометрических примитивов с артефактами сшивки для последующего выравнивания.

Бесшовное смешивание изображений, как правило, является необходимым после построения панорамного снимка в связи с возникающими локальными артефактами освещенности, рассогласования текстурной мозаики и т.д. Известны четыре категории методов, а именно: дискретные цветовые методы, методы на основе построения пирамиды изображений, градиентные методы и многополосное смешивание [14]. Многополосное смешивание относится к высокоэффективным, но наиболее затратным методам устранения артефактов сшивки. Идея метода, предложенного в [15], заключается в смешивании низких частот по большому пространственному диапазону, а высоких частот - по малому пространственному диапазону. Результатом многополосного смешивания является суммирование изображений по всем поддиапазонам.

\section{Описание экспериментального исследования}

Для проведения экспериментов использовался набор изображений Swiss dataset [16], полученный дроном еВеe RTK, имеющим следующие характеристики: масштаб - 4 см/пикс., область - 0,18 км², высота полета - 169 м, число изображений - 32 . Пример создания панорамного аэрофотоснимка на основе многополосного смешивания для набора из 32 изображений представлен на рисунке 4.

Тестирование проводилось на компьютере со следующей конфигурацией: процессор AMD Athlon II X3 $3.2 \mathrm{GHz}$, оперативная память 8GB DDR3 1333Mhz, видеокарта NVIDIA GeForce GTX 650. В таблице 1 представлено время работы алгоритма нахождения особых точек, преобразования и сопоставления изображений с разным разрешением при использовании алгоритмов FAST, FASTER и SURF. Сравнение времени работы алгоритмов расчета матрицы гомографии при сопоставлении изображений с разрешением $1280 \times 720$ пикселов приведено в таблице 2.

Таблииа 1

Время работы алгоритма сопоставления изображений (мс)

Table 1

The execution time of image matching algorithm (ms)

\begin{tabular}{|c|c|c|c|}
\hline $\begin{array}{c}\text { Разрешение, } \\
\text { пиксел }\end{array}$ & SURF & FAST-ER & FAST \\
\hline $640 \times 360$ & 535,34 & 22,05 & 13,11 \\
\hline $852 \times 480$ & 782,27 & 33,58 & 18,97 \\
\hline $1280 \times 720$ & 1380,11 & 65,81 & 29,34 \\
\hline $1600 \times 900$ & 1965,59 & 90,63 & 34,47 \\
\hline $1920 \times 1080$ & 2844,87 & 132,55 & 43,67 \\
\hline
\end{tabular}




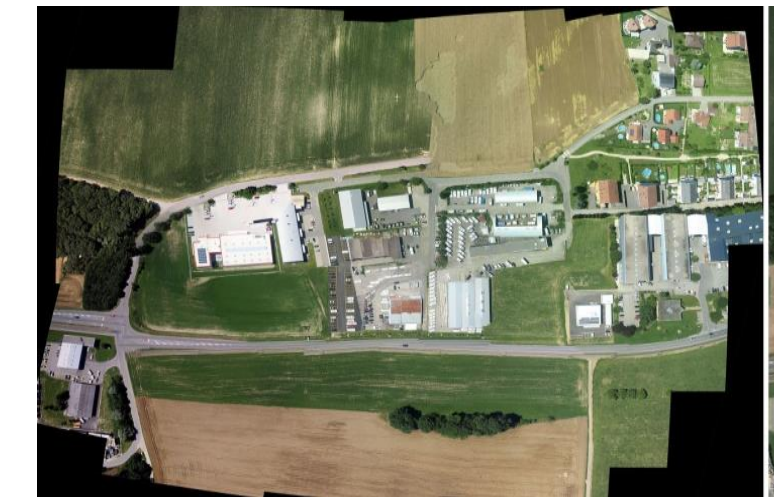

a)

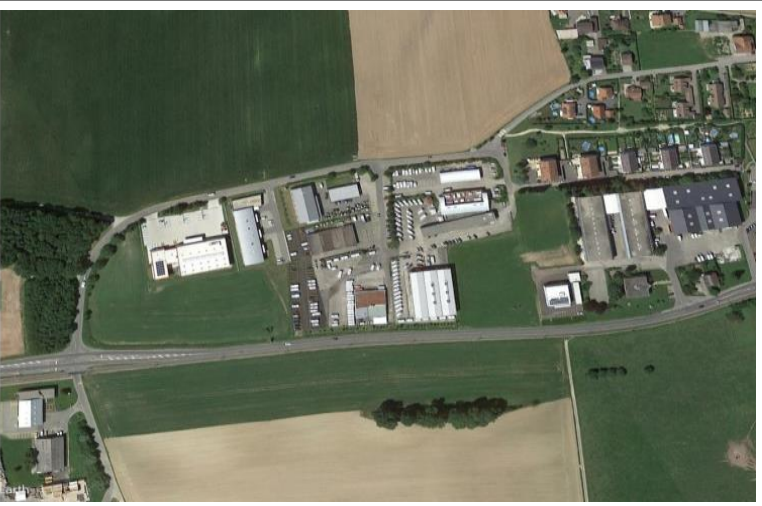

б)

Puc. 4. Панорамный аэрофотоснимок в сравнении с изображением Google Maps: a) панорамный аэрофотоснимок, б) изображение с Google Maps

Fig. 4. Panoramic aerial image in comparison with the Google Maps image: a) panoramic aerial image, б) Google Maps image

Таблица 2

Сравнение времени работы (мс) алгоритмов RANSAC и MLESAC

Comparison of the operating time of RANSAC and MLESAC algorithms (ms)

\begin{tabular}{|l|c|c|c|}
\hline Алгоритм & FAST & FAST-ER & SURF \\
\hline RANSAC & 2,01 & 1,17 & 3,55 \\
\hline MLESAC & 2,29 & 1,35 & 4,09 \\
\hline
\end{tabular}

Проведенные исследования показали, что использование алгоритма MLESAC позволяет повысить точность сопоставления особых точек в среднем на $5 \%$ по сравнению с алгоритмом RANSAC, при этом скорость работы алгоритма уменьшается в среднем на $15 \%$.

\section{Заключение}

Применение алгоритмов FAST и FAST-ER позволяет организовать сопоставление изображений (с разрешением до 1920×1080 пикселов) для формирования панорамного снимка в реальном режиме времени, а слияние изображений с применением технологии многополосного смешивания обеспечивает качественную визуализацию в местах сшивки. Основным недостатком рассмотренных алгоритмов является использование в расчетах яркостной составляющей пиксела, что может приводить к некорректным результатам сшивки при наличии на изображениях областей с повышенной яркостью или больших фрагментов, содержащих блики.

Работа выполнена при финансовой поддержке РФФИ, грант № 16-07-00121 A.

\section{Лumepamypa}

1. He K., Chang H., Sun J. Rectangling panoramic images via warping. Proc. Conf. ACM Transactions on Graphics - SIGGRAPH, 2013, vol. 32 , i. 4 , art. 79
2. Bold S., Sosorbaram B., Lee S.R. Implementation of autonomous unmanned aerial vehicle with moving-object detection and face recognition. Information Science and Applications (ICISA). 2016, vol. 376, pp. 361-370.

3. Буряченко В.В., Фаворская М.Н., Зотин А.Г., Пахирка А.И. Восстановление границ кадра при стабилизации на основе построения модели фона и оценки значимости объектов // Информационно-управляющие системы. 2017. № 5. С. 42-51.

4. Leutenegger S., Chli M., Siegwart R.Y. BRISK: Binary robust invariant scalable keypoints. Proc. IEEE Intern. Conf. Computer Vision (ICCV), 2011, pp. 2548-2555.

5. Bay H., Ess A., Tuytelaars T., van Gool L. Speeded-up robust features (SURF). Computer Vision and Image Understanding. 2008, vol. 110 , no. 3, pp. 346-359.

6. Lowe D.G. Distinctive image features from scale-invariant keypoints. Intern. J. of Computer Vision. 2004, vol. 60, no. 2, pp. 91-110.

7. Tuytelaars T., van Gool L.J. Matching widely separated views based on affine invariant regions. Intern. J. of Computer Vision. 2004, vol. 59, no. 1, pp. 61-85.

8. Civera J., Davison A.J., Magallón J.A., Montiel J.M. Driftfree realtime sequential mosaicing. Intern. J. of Computer Vision. 2009 , vol. 81 , no. 2 , pp. $128-137$.

9. Зотин А.Г., Пахирка А.И., Дамов М.В. Сравнение алгоритмов получения точечных особенностей для системы сопоставления кадров видеопоследовательности // Региональные проблемы дистанционного зондирования Земли: матер. IV Междунар. науч. конф. 2017. С. 112-115.

10. Quinlan J.R. Induction of decision trees. Machine Learning. 1986, vol. 1, pp. 81-106.

11. Rublee E., Rabaud V., Konolige K., Bradski G. ORB: an efficient alternative to SIFT or SURF Proc. IEEE Intern. Conf. Computer Vision (ICCV), 2011, pp. 2564-2571.

12. Fischler M.A., Bolles R.C. Random Sample Consensus: A Paradigm for Model Fitting with Applica-tions to Image Analysis and Automated Cartography. Communications of the ACM, 1981, vol. 24 , no. 6 , pp. 381-395

13. Torr P.H.S., Zisserman A. MLESAC: A New Robust Estimator with Application to Estimating Im-age Geometry. Computer Vision and Image Understanding, 2000, vol. 78, pp. 138-156.

14. Favorskaya M.N., Buryachenko V.V., Zotin A.G., Pakhirka A.I. Video completion in digital stabili-zation task using pseudopanoramic technique. Proc. 2nd International ISPRS, 2017, pp. 83-90.

15. Burt P.J., Adelson E.H. A multiresolution spline with application to image mosaics. ACM Transac-tions on Graphics, 1983, vol. 2, no. 4, pp. 217-236.

16. Example Datasets. URL: https://www.sensefly.com/drones/ example-datasets.html (дата обращения: 14.11.2017). 


\section{CREATING PANORAMIC AERIAL IMAGES FROM QUADCOPTER}

A.I. Pakhirka ${ }^{1}$, Ph.D. (Engineering), Associate Professor, pahirka@sibsau.ru A.G. Zotin ${ }^{1}$, Ph.D. (Engineering), Associate Professor, zotinkrs@gmail.com

V.V. Buryachenko ${ }^{1}$, Ph.D. (Engineering), Associate Professor, buryachenko@sibsau.ru

${ }^{1}$ Academician M.F. Reshetnev Siberian State University of Science and Technology, Krasnoyarsky Rabochy Ave. 31, Krasnoyarsk, 660037, Russian Federation

Abstract. The paper considers the approach to forming panoramic images representing highly detailed images of some area. To obtain high-quality panoramic images, there is a need in specialized equipment and, if possible, the maximum survey altitude. These factors can be excluded if stitching the images from cameras of inexpensive unmanned aerial vehicles, i.e. quadcopters. One of the approaches to forming panoramic images is using a set of images or video sequence data obtained when surveying by a quadcopter or other unmanned aerial vehicle.

Images are stitched by the methods of matching point features. However, feature points detection algorithms should ensure invariance to any transformations of an image in order to analyze data and generate panoramic images. The research includes applying such algorithms such as FAST, FAST-ER and SURF due to the fact that they are currently the most common solutions for such task. The algorithms for determining correspondences of found feature points are RANSAC and MLESAC.

The paper proposes the algorithm of creating a panoramic aerial image from a set of successive landscape images from an unmanned aerial vehicle based on matching key features. The authors also use panoramic image global adjustment through affine processing. A special attention is paid to stitching images using multi-band blending techniques. This provides highquality visualization in the stitching places of a panoramic aerial image.

Keywords: feature point detectors, FAST, SURF, panoramic aerial image.

Acknowledgements. The work has been financially supported by RFBR, grant no. 16-07-00121 A.

\section{References}

1. He K., Chang H., Sun J. Rectangling panoramic images via warping. ACM Trans. on Graphics - SIGGRAPH 2013 Conf. 2013, vol. 32, i. 4, art. 79

2. Bold S., Sosorbaram B., Lee S.R. Implementation of autonomous unmanned aerial vehicle with moving-object detection and face recognition. Information Science and Applications (ICISA). 2016, vol. 376, pp. 361-370.

3. Buryachenko V.V., Favorskaya M.N., Zotin A.G., Pakhirka A.I. Restoration of frame borders under stabilization based on background model building and salient objects estimation. Informatsionno-upravlyayushchie sistemy [Information and Control Systems]. 2017, vol. 90, pp. 42-51 (in Russ.).

4. Leutenegger S., Chli M., Siegwart R.Y. BRISK: Binary robust invariant scalable keypoints. IEEE Int. Conf. Computer Vision (ICCV). 2011, pp. 2548-2555.

5. Bay H., Ess A., Tuytelaars T., Van Gool L. Speeded-up robust features (surf). Computer Vision and Image Understanding. 2008, vol. 110 , no. 3, pp. 346-359.

6. Lowe D.G. Distinctive image features from scale-invariant keypoints. Int. Jour. of computer vision. 2004, vol. 60, no. 2, pp. 91-110.

7. Tuytelaars T., Van Gool L.J. Matching widely separated views based on affine invariant regions. Int. Jour. of Computer Vision. 2004, vol. 59, no. 1, pp. 61-85.

8. Civera J., Davison A.J., Magallón J.A., Montiel J.M. Drift-free realtime sequential mosaicking. Int. Jour. on Computer Vision. 2009, vol. 81, no. 2, pp. 128-137.

9. Zotin A.G., Pakhirka A.I., Damov M.V. Feature detection algorithms comparison for video frames matching system. Regionalnye problemy distantsionnogo zondirovaniya Zemli: mater. IV Mezhdunar. nauch. konf. [Proc. 4th Int. Sci. Conf. Regional Problems of Earth Remote Sensing]. Krasnoyarsk, 2017, pp. 112-115 (in Russ.).

10. Quinlan J.R. Induction of decision trees. Machine Learning. 1986, vol. 1, pp. 81-106.

11. Rublee E., Rabaud V., Konolige K., Bradski G. ORB: an efficient alternative to SIFT or SURF. IEEE Int. Conf. on Computer Vision (ICCV). 2011, pp. 2564-2571.

12. Fischler M.A., Bolles R.C. Random Sample Consensus: A Paradigm for Model Fitting with Applications to Image Analysis and Automated Cartography. Communications of the ACM. 1981, vol. 24, no. 6, pp. 381-395.

13. Torr P.H.S., Zisserman A. MLESAC: A new robust estimator with application to estimating image geometry. Computer Vision and Image Understanding. 2000, vol. 78, pp. 138-156.

14. Favorskaya M.N., Buryachenko V.V., Zotin A.G., Pakhirka A.I. Video completion in digital stabilization task using pseudo-panoramic technique. Proc. 2nd International ISPRS. 2017, pp. 83-90.

15. Burt P.J., Adelson E.H. A multiresolution spline with application to image mosaics. ACM Trans. on Graphics. 1983, vol. 2 , no. 4 , pp. $217-236$ 2017)

16. Example Datasets. Available at: https://www.sensefly.com/drones/example-datasets.html (accessed November 14, 